\title{
Nanoparticle Motion in Entangled Melts of Linear and Nonconcatenated Ring Polymers
}

\author{
Ting Ge, ${ }^{\dagger}$ Jagannathan T. Kalathi, ${ }^{\ddagger}$ Jonathan D. Halverson, ${ }^{\S}$ Gary S. Grest, ${ }^{\|}$and Michael Rubinstein*, ${ }^{\dagger}$ \\ ${ }^{\dagger}$ Department of Chemistry, University of North Carolina, Chapel Hill, North Carolina 27599, United States \\ ${ }^{\ddagger}$ Department of Chemical Engineering, National Institute of Technology Karnataka, Surathkal, Mangalore 575025, India \\ ${ }^{\S}$ Max Planck Institute for Polymer Research, Ackermannweg 10, 55128 Mainz, Germany \\ "Sandia National Laboratories, Albuquerque, New Mexico 87185, United States
}

\section{Supporting Information}

ABSTRACT: The motion of nanoparticles (NPs) in entangled melts of linear polymers and nonconcatenated ring polymers are compared by large-scale molecular dynamics simulations. The comparison provides a paradigm for the effects of polymer architecture on the dynamical coupling between NPs and polymers in nanocomposites. Strongly suppressed motion of NPs with diameter $d$ larger than the entanglement spacing $a$ is observed in a melt of linear polymers before the onset of Fickian NP diffusion. This strong suppression of NP motion occurs progressively as $d$ exceeds $a$ and is related to the hopping diffusion of NPs in the entanglement network. In contrast to the NP motion in linear polymers, the motion of NPs with $d>a$ in ring polymers is not as strongly suppressed prior to Fickian diffusion. The diffusion coefficient $D$ decreases with increasing $d$ much slower in entangled rings than in entangled linear chains. NP motion in entangled nonconcatenated ring polymers is understood through a scaling analysis of the coupling between NP motion and the self-similar entangled dynamics of ring polymers.

\section{INTRODUCTION}

Polymer nanocomposites that integrate nanoparticles (NPs) into polymer melts often possess superior mechanical, thermal, optical, or electrical properties in comparison with pure polymeric materials. ${ }^{1}$ Fabrication and processing of polymer nanocomposites require a good understanding of their viscoelastic behavior. ${ }^{2-4}$ Central to the viscoelasticity of polymer nanocomposites is the coupling between the motion of NPs and the relaxation dynamics of matrix chains.

The dynamical coupling between NPs and linear polymers depends on the particle diameter $d$ compared to the polymer entanglement mesh size $a$, as shown in experiments, ${ }^{5-8}$ simulations, ${ }^{4,9,10}$ and theoretical studies. ${ }^{11-16}$ For $d<a$, the motion of NPs is coupled to the unentangled dynamical modes of local chain segments up to sizes $\approx d$. This coupling gives rise NPs with $d>a$ are confined by the entanglement mesh for time scales greater than the relaxation time $\tau_{\mathrm{e}}$ of an entanglement strand, as illustrated in Figure 1a. Brochard-Wyart and de Gennes $^{11}$ argued that NPs with $d>a$ have to wait for the complete relaxation of surrounding entangled polymers to diffuse. However, Cai et al. ${ }^{14}$ recently proposed a hopping diffusion mechanism for NPs moderately larger than $a$ to overcome the confinement by the entanglement network. A qualitatively different activated hopping mechanism for NPs to to subdiffusive motion prior to Fickian diffusion. In contrast,
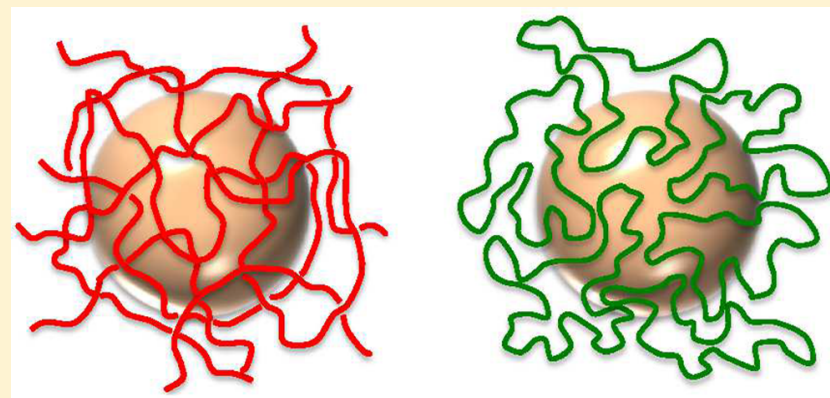

a

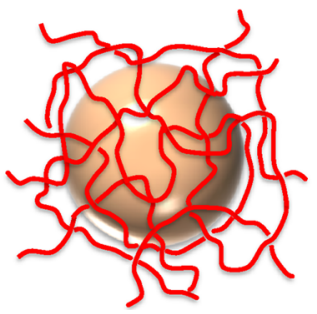

b

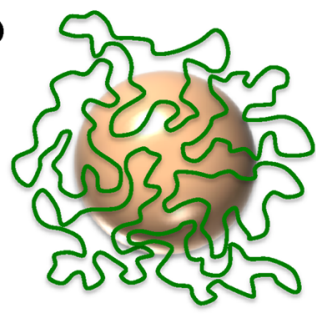

Figure 1. Schematic sketch of a NP (light orange sphere) in (a) entangled linear polymers (red lines) and (b) entangled nonconcatenated ring polymers (green lines).

overcome confinement is described in a nonlinear Langevin equation theory for NP motion in cross-linked networks and entangled melts of polymers. ${ }^{15}$ Microscopic force-level theories ${ }^{13,16}$ without considering the mechanism of NP hopping also predict a partial coupling between NPs and the entanglement network for $d$ moderately larger than $a$.

The discrepancy between the dynamics of NPs smaller and larger than the entanglement mesh size in linear polymer melts reflects the impact of entanglements on NP motion.

Received: December 6, 2016

Revised: January 20, 2017

Published: February 13, 2017 
Entanglements also play an important role in the viscoelasticity of high molecular weight polymers with nonlinear architectures, such as ring, star, and branched polymers. ${ }^{17,18}$ One would anticipate intriguing dynamical couplings between NPs and entangled polymers with nonlinear architectures. We study the motion of NPs in entangled nonconcatenated ring polymers as illustrated in Figure $1 \mathrm{~b}$. Ring polymers are particularly interesting as their unique topology gives rise to distinctive conformational and dynamic properties. ${ }^{19-30}$ Entangled nonconcatenated ring polymers adopt compact conformations ${ }^{20,22}$ rather than the almost Gaussian random-walk conformations of linear polymers. The entanglements in ring polymers do not create long-lived tubes that confine polymer motion as in entangled linear polymers. The entangled dynamics of ring polymers is self-similar, and there is a power-law time dependence of the stress relaxation function without the rubbery plateau characteristic of entangled linear polymers. ${ }^{18}$ Nonconcatenated entangled ring polymers are good models of the chromatin packed in cell nucleus ${ }^{31,32}$ and of the strands of deswollen gel networks. ${ }^{33}$

\section{MODEL AND METHODOLOGY}

Molecular dynamics (MD) simulations of NPs in melts of entangled nonconcatenated ring polymers and entangled linear polymers are performed. The models of polymers and NPs are similar to those in previous simulations. ${ }^{4,10,20,21,34,35}$ The beadspring model ${ }^{34}$ is employed to simulate the polymers. All beads of size $\sigma$ and mass $m$ interact via the Lennard-Jones (LJ) potential with interaction strength $\epsilon$, cutoff distance $r_{\mathrm{c}}=2.5 \sigma$, and characteristic time $\tau=\sigma \sqrt{m / \epsilon}$. Neighboring beads in a polymer are connected additionally by the finitely extensible nonlinear elastic (FENE) potential. A bond bending potential $V_{\theta}=k_{\theta}(1+\cos \theta)$ is applied with $k_{\theta}=1.5 \epsilon$. NPs are modeled as smooth spheres that interact with each other through a purely repulsive potential. ${ }^{4,10,35,36}$ The mass density of a NP is $\rho=m / \sigma^{3}$. Interactions between NPs and polymers are weakly attractive, so NPs are well dispersed in polymers.

An entangled melt of $M=200$ linear polymers was simulated. For the linear polymers, the number of monomers per entanglement strand $N_{\mathrm{e}} \approx 28$, $^{37-39}$ the number of monomers per chain $N=800 \approx 30 N_{\mathrm{e}}$, the average spacing between entanglements $a \approx 5 \sigma,{ }^{38}$ and the entanglement time $\tau_{\mathrm{e}} \approx$ $4000 \tau .{ }^{40} \mathrm{We}$ simulated two entangled melts of ring polymers. One contains $M=200$ rings with $N=800$ monomers per ring. The other has $M=1600$ rings with $N=100$. The melt density is $\rho_{\text {melt }}=0.89 \mathrm{~m} / \sigma^{3}$ for both ring and linear polymers. The rootmean-square end-to-end distance of the linear polymers is $R_{\mathrm{e}} \approx$ 41.2 $\sigma$. The root-mean-square spanning distance of ring polymers between pairs of beads separated by $N / 2$ beads is $R$ $\approx 15.3 \sigma$ and $7.0 \sigma$ for $N=800$ and 100 , respectively.

The samples of NPs in linear polymers were built using the same method in previous simulations by Kalathi et al. ${ }^{40}$ The samples of NPs in ring polymers were made by inserting NPs into well-equilibrated melts of nonconcatenated ring polymers from previous simulations by Halverson et al. ${ }^{20,21}$ The insertion of NPs into ring polymers started with creating well-separated small voids with volumes $\approx \sigma^{3}$ in a ring polymer melt. Then the volumes of the voids were increased until each void was large enough to accommodate a NP. During the insertion, a few pairs of rings became concatenated. One ring of each concatenated pair was removed from the simulation box to ensure nonconcatenation. NP diameter $d$ ranges from $3 \sigma<a$ to $15 \sigma$ $\approx 3 a$. The volume fraction of NPs $\approx 10 \%$. It is sufficiently low for NP motion to be barely affected by the interactions between NPs, while the corresponding number of NPs $N_{\mathrm{NP}}$ is sufficiently large for good statistics of $\mathrm{NP}$ motion. $N_{\mathrm{NP}}$ ranges from 650 for $d=3 \sigma$ to 5 for $d=15 \sigma$. We also simulated monomers with $d=1 \sigma$ dispersed in polymer melts at volume fraction $10 \%$. All samples were equilibrated at pressure $P=0$ and temperature $T=1.0 \epsilon / k_{\mathrm{B}}$. Subsequent simulations were run at constant volume $V$ for up to $10^{8} \tau$ depending on $d$.

\section{RESULTS AND DISCUSSION}

3.1. NPs in Linear Polymers. The mean-square displacements (MSDs) of NPs in entangled ring and linear polymer melts are compared in Figure 2a. For NPs in linear chains, the smallest NPs with $d=3 \sigma<a$ move subdiffusively with the minimum log-log slope of MSD $\alpha_{\text {min }} \approx 0.7$. The crossover to Fickian diffusion with $\alpha=1$ occurs at ${ }^{40} t<\tau_{\mathrm{e}} \approx 4000 \tau$ (see dashed black line in Figure 2a). Larger NPs with $d \geq a \approx 5 \sigma$ also move subdiffusively after the ballistic regime, but the crossover to Fickian diffusion occurs at $t>\tau_{e}$ (see dashed color lines in Figure 2a). $\alpha_{\text {min }}$ decreases from $\approx 0.58$ for $d=5 \sigma$ to $\approx 0.14$ for $d=15 \sigma$, as shown in the inset of Figure $2 \mathrm{a}$.

For NPs in linear chains, the subdiffusion of NPs with $d<a$ and the subdiffusion of NPs with $d>a$ for $t<\tau_{\mathrm{e}}$ arise from the coupling between NP motion and the unentangled dynamics of chain segments. The theoretical prediction is $\alpha=1 / 2$ based on the Rouse model of unentangled polymer dynamics. ${ }^{11,12}$ This predicted asymptotic scaling regime is not well developed in the present simulations. For $d<a, \alpha$ in the subdiffusive regime is affected by the crossover to the Fickian regime. For $d>a, \alpha$ in the subdiffusive regime for $t<\tau_{\mathrm{e}}$ is affected by the crossover to the regime for $t>\tau_{\mathrm{e}}$.

The motion of NPs with $d>a$ in linear chains is further suppressed for $t>\tau_{\mathrm{e}}$ because of confinement by the entanglement network. While the theory by Brochard-Wyart and de Gennes ${ }^{11}$ predicts that $\alpha_{\min }$ decreases to 0 sharply at $d \approx$ $a, \alpha_{\min }$ in the simulations progressively decreases with $d$ over a broad range up to $d \approx 3 a$ (see the inset of Figure $2 \mathrm{a}$ ). For $d$ moderately larger than $a$, NPs can overcome the confinement of the entanglement network through the hopping diffusion mechanism. ${ }^{14}$ In hopping diffusion theory, the entanglement network is described as many overlapping yet independent elementary networks, the centers of which are separated by an average distance $\approx \sigma$. Each hop of a NP confined by elementary networks corresponds to the displacement from one network cage to a neighboring cage over a distance $\approx \sigma$. A successful hop needs to overcome a free energy barrier associated with the fluctuation of one network strand from the average size $a$ to $d$. The barrier height is proportional to $d / a$, and therefore as $d$ increases the probability of hopping decreases as $\exp (-d / a)$. A qualitatively different picture of activated NP hopping is presented in a microscopic force-level theory. ${ }^{15}$ It predicts that hopping distance of a large NP in an entangled polymer melt increases with $d$, and the hopping barrier height is asymptotically proportional to the volume of a NP. An alternative microscopic force-level theory ${ }^{13,16}$ excludes the activated NP hopping.

As hopping becomes less likely with increasing $d$, the confinement of the entanglement network is enhanced, and $\alpha_{\min }$ is reduced. For $d \gg a$, hopping diffusion is highly unlikely, and NPs have to wait for the relaxation of the entanglement network to diffuse. In this case, $\alpha_{\min }$ would be independent of $d$. However, $\alpha_{\min }$ is expected to be nonvanishing for $N / N_{\mathrm{e}} \approx 30$ 
a
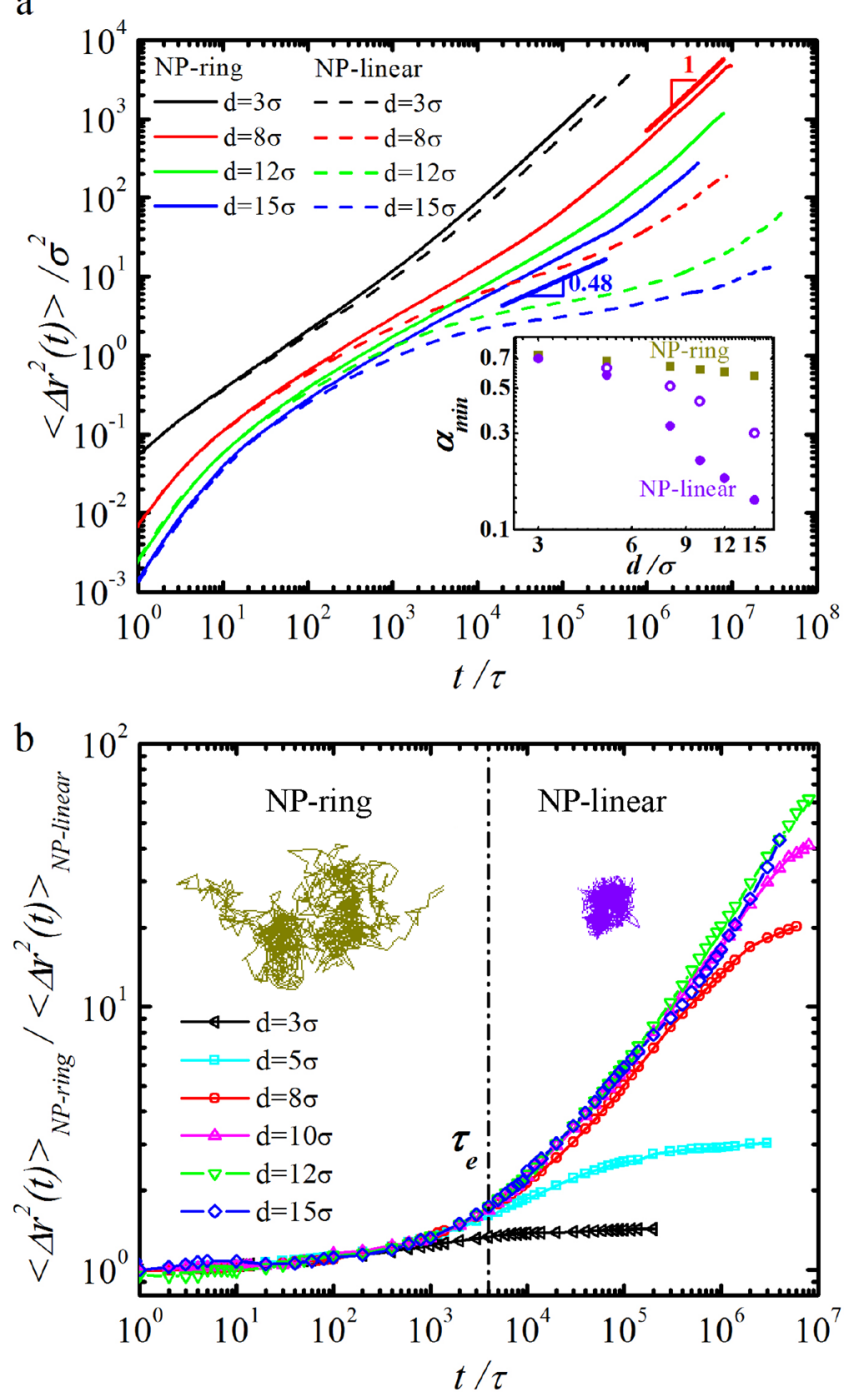

Figure 2. (a) Mean-square displacements $\left\langle\Delta r^{2}(t)\right\rangle$ of NPs with sizes $d$ $=3 \sigma-15 \sigma$ in entangled melts of nonconcatenated ring polymers (solid lines) and linear polymers (dashed lines) with $N=800$. Thick red line indicates the $\log -\log$ slope $\alpha=1$ for Fickian diffusion. Thick blue line indicates the expected $\alpha \approx 0.48$ for complete dynamical coupling between NPs and rings. The inset shows $\alpha_{\min }$ vs $d$ for NP-linear (filled circles) and NP-ring (filled squares) systems and for NP-linear systems (open circles) in previous simulations ${ }^{10}$ with smaller $N / N_{\mathrm{e}} \approx 9$ and larger entanglement mesh size $a \approx 7 \sigma$. (b) Ratio of the MSDs of NPs in nonconcatenated rings $\left\langle\Delta r^{2}(t)\right\rangle_{\mathrm{NP}-\text { ring }}$ to the corresponding MSDs in linear chains $\left\langle\Delta r^{2}(t)\right\rangle_{\text {NP-linear }}$ Dash-dotted line indicate the entanglement time $\tau_{\mathrm{e}} \approx 4000 \tau \tau^{40}$ The insets illustrate the trajectories of a NP with $d=10 \sigma$ in entangled rings (dark yellow line) and linear chains (violet line) for a time period of $10^{5} \tau \approx 25 \tau_{\mathrm{e}}$.

because the entanglement network can partially relax through longitudinal Rouse modes in a confining tube, tube length fluctuation, and constraint release. ${ }^{41}$

Hopping events are not directly observable in the trajectories of NPs for $a<d<a^{2} / \sigma{ }^{14}$ as they are masked by the thermal fluctuations of NPs confined by individual cages. The MSD due to thermal fluctuation of a NP confined in an entanglement cage is $\left\langle\Delta r^{2}\right\rangle_{\text {fluct }} \approx \sigma^{2} a^{2} /(d \sigma)$. For $a<d<a^{2} / \sigma,\left\langle\Delta r^{2}\right\rangle_{\text {fluct }}$ is larger than $\sigma^{2}$ resulting from one hopping event. For $d \geq a^{2} / \sigma$, $\left\langle\Delta r^{2}\right\rangle_{\text {fluct }}<\sigma^{2}$, but the propability of hopping $\sim \exp (-d / a)$ is lower.
The contribution of hopping diffusion to the MSD is manifested in the dependence of $\alpha_{\min }$ on $d$. Kalathi et al. ${ }^{10}$ have simulated NPs in linear polymers with $k_{\theta}=0.75 \epsilon, N_{\mathrm{e}} \approx 45, N=$ $400 \approx 9 N_{\mathrm{e}}$, and $a \approx 7 \sigma$. The dependence of $\alpha_{\min }$ on $d$ for their simulations is shown in the inset of Figure $2 \mathrm{a}$ by the open circles. Compared to the previous simulations, the present simulations with $N / N_{\mathrm{e}} \approx 30$ and $a \approx 5 \sigma$ enable the exploration of hopping diffusion over a broader range of $d / a$, as the largest $d / a$ is increased from $\approx 2$ to $\approx 3$. Higher $N / N_{\mathrm{e}}$ is favorable because the reptation time $\sim\left(N / N_{\mathrm{e}}\right)^{3.4}$ is longer, and thus the contribution to NP mobility from hopping diffusion is more dominant over the competing contribution from chain reptation. ${ }^{14} \alpha_{\min }$ at the largest $d / a \approx 0.14$ in the present simulations is reduced with respect to $\approx 0.3$ in the previous simulations. To our knowledge, this is the first time such a strongly suppressed motion of NPs in entangled linear polymer melts is observed in simulations.

3.2. NPs in Ring Polymers. NPs in entangled nonconcatenated ring polymers differ from their counterparts in linear chains. For $d=3 \sigma, \mathrm{NP}$ motion in a melt of rings is comparable to but slightly faster than in a melt of linear chains. The ratio of the MSDs in rings and linear chains is $\approx 1.4$ in the Fickian regime (see Figure $2 b$ ). More remarkable differences between MSDs in entangled ring and linear polymers are observed for $d \geq a \approx 5 \sigma . \alpha_{\min }$ for NPs in rings decreases slowly with $d$ and is $\approx 0.6$ for $d=15 \sigma$ (see the inset of Figure 2a). Unlike in entangled linear chains, the MSDs are not strongly suppressed in entangled rings even for the largest $d$. The ratio of the MSDs in rings and linear chains is already $\approx 2$ at $t \approx \tau_{\mathrm{e}}$ and increases with $t$ for $t>\tau_{\mathrm{e}}$ as shown in Figure $2 \mathrm{~b}$. The trajectories of a NP with $d=10 \sigma>a$ during a time period $10^{5} \tau$ $\approx 25 \tau_{\mathrm{e}}$ in entangled rings and linear chains are illustrated in the inset of Figure $2 \mathrm{~b}$. The absence of strongly suppressed NP motion in entangled rings demonstrates that there are no longlived confining tubes in entangled nonconcatenated rings at least for $N / N_{\mathrm{e}}$ up to $\approx 30$, consistent with experiments ${ }^{19,23}$ and simulations $^{21}$ of entangled ring melts.

To understand the subdiffusive motion of NPs in ring polymers, we developed a scaling model for the dynamical coupling between NPs and entangled rings. Entangled ring polymers relax in a self-similar manner for $t>\tau_{\mathrm{e}}$ through relaxations of progressively larger sections until the entire rings of size $R$ are relaxed. ${ }^{27}$ The motion of NPs with size $a<d<R$ is coupled to the relaxations of ring sections with sizes up to $d$. This coupling gives rise to the subdiffusive motion of NPs for $\tau_{\mathrm{e}}<t<\tau_{\mathrm{s}}$, where $\tau_{\mathrm{s}}$ is the relaxation time of a ring section with size $\approx d$. For $\tau_{\mathrm{e}}<t<\tau_{\mathrm{s}}$, a ring section that has just relaxed contains $g$ monomers. The scaling ansatz is that the effective viscosity $\eta_{\text {eff }}$ experienced by a NP at $t$ is the same as the melt viscosity of ring polymers with $N=g$.

Annealed lattice tree model ${ }^{26}$ and fractal loopy globule (FLG) model ${ }^{27}$ have been proposed to describe the dynamics of multiple entangled nonconcatenated ring polymers. We use the FLG model that self-consistently accounts for the complete tube dilation with an appropriate renormalization of monomeric friction at different time scales. ${ }^{27}$ In the FLG model, the conformation of a ring polymer is described as a hierarchy of entangled loops. The loops at different length scales overlap with each other in a self-similar manner such that the overlap parameter is the same at all scales and equal to the KavassalisNoolandi number ${ }^{42} \mathrm{O}_{\mathrm{KN}} \approx 20$ for the onset of entanglements. The constant overlap parameter at the entanglement threshold $O_{\mathrm{KN}}$ for length scales above the entanglement spacing has been 
confirmed in the simulations by Rosa and Everaers. ${ }^{25}$ During the relaxation of ring polymers, topological constraints associated with rearranged smaller loops affect the dynamics of larger loops through increasing the effective friction coefficient. Nevertheless, these topological constraints do not contribute to the tubes confining the larger loops and thus are dynamically released. The tube diameter as the average spacing between dynamically relevant topological constraints increases with time and is comparable to the size of a characteristic loop that has just been rearranged. This picture of complete tube dilation self-consistently describes the dynamics of entangled rings without long-lived tubes. The FLG model predicts that the relaxation time of a ring section containing $g$ monomers scales as $\tau_{\text {relax }}(g) \approx \tau_{\mathrm{e}}\left(g / N_{\mathrm{e}}\right)^{7 / 3}$. Note that the exponent $7 / 3$ is between 2 for Rouse dynamics of unentangled polymers and 3 for reptation dynamics of entangled linear polymers.

According to the FLG model, the number of monomers in a relaxed ring section increases with time as $g \sim t^{3 / 7}$. The terminal relaxation time for a ring with $N$ monomers is $\tau_{\text {relax }} \approx \tau_{\mathrm{e}}\left(N / N_{\mathrm{e}}\right)^{7 / 3}$. The stress relaxation modulus $G\left(\tau_{\text {relax }}\right) \approx$ $k_{\mathrm{B}} T / v N$ at $t \approx \tau_{\text {relax }}$ is on the order of thermal energy $k_{\mathrm{B}} T$ per volume $v N$ of a ring polymer, where $v$ is the volume of a monomer. As a result, the melt viscosity of ring polymers $\eta \approx$ $\tau_{\text {relax }} G\left(\tau_{\text {relax }}\right) \sim N^{7 / 3} / N \sim N^{4 / 3}$. Using $g \sim t^{3 / 7}$ and $\eta \sim N^{4 / 3}$, we obtain $\eta_{\text {eff }} \sim g^{4 / 3} \sim t^{4 / 7}$ for $\tau_{\mathrm{e}}<t<\tau_{\mathrm{s}}$. The effective diffusion coefficient $D_{\text {eff }} \sim k_{\mathrm{B}} T /\left(\eta_{\text {eff }} d\right) \sim t^{-4 / 7}$, and thus $\left\langle\Delta r^{2}(t)\right\rangle \sim D_{\text {eff }} t$ $\sim t^{3 / 7}$ for $\tau_{\mathrm{e}}<t<\tau_{\mathrm{s}}$. The resulting scaling exponent for the power-law subdiffusion of NPs in entangled rings is $\alpha=3 / 7 \approx$ 0.43 . Recently, Grosberg et al. ${ }^{43}$ have theoretically studied the scale-dependent viscosity in polymer fluids. For the FLG model of nonconcatenated ring polymer melts, they obtained $\eta(\omega) \sim$ $\omega^{-4 / 7}$ for $1 / \tau_{\text {relax }}<\omega<1 / \tau_{\mathrm{e}}$. Since $\omega \sim 1 / t$, this agrees with our result $\eta_{\text {eff }} \sim t^{4 / 7}$.

The MSD subdiffusive exponent $\alpha$ is identical to the exponent $\beta$ for the power-law time dependence of the stress relaxation modulus associated with the self-similar dynamics of entangled rings. Because of the finite polymer size, the relaxation time of ring polymers is not well separated from $\tau_{\mathrm{e}}$ even for $N / N_{\mathrm{e}} \approx 30$, and thus the asymptotic entangled dynamics is not well developed. Previous analysis ${ }^{27}$ has shown that $\beta \approx 0.48$ for $N / N_{\mathrm{e}} \approx 30$, and the asymptotic theoretical value $3 / 7 \approx 0.43$ could only be observed for $N / N_{\mathrm{e}}>100$. For NPs in rings with $N / N_{\mathrm{e}} \approx 30$, it is expected that $\alpha \approx \beta \approx 0.48$ for sufficiently large NPs that can be completely coupled to all the relaxation modes of rings.

Simulation results of $\alpha_{\text {min }}$ for NPs in ring polymers (see the inset of Figure $2 \mathrm{a}$ ) are all above the expected value 0.48 (as indicated by the thick blue line in Figure $2 \mathrm{a}$ ) for $N / N_{\mathrm{e}} \approx 30$. This is attributed to the finite sizes of NPs, which are not large enough to allow the complete dynamical coupling between NPs and relaxation modes of rings. The effects of $d$ on the dynamical coupling between NPs and rings can be quantified by the dependence of $\alpha_{\min }$ on $d . \alpha_{\min }$ decreases as $d$ increases, indicating that the degree of the dynamical coupling increases with $d$. Extrapolation of the $d$ dependence of $\alpha_{\text {min }}$ yields that the expected value 0.48 for complete coupling would occur for $d>$ $d_{\mathrm{c}} \approx 45 \sigma . d_{\mathrm{c}}$ is about 2.6 times the root-mean-square average spanning distance $R \approx 15.3 \sigma$ between pairs of monomers separated by $N / 2$ monomers (see Figure $S 1$ ). The exact value of $d_{\mathrm{c}} / R$ may be related to the shape of NPs and the boundary conditions between NPs and polymers. Future work is needed to elucidate the factors affecting $d_{\mathrm{c}} / R$.
3.3. Fickian Diffusion of NPs. As shown in Figure 2a, the NPs have reached the Fickian diffusive regime except for the largest NPs in linear chains. Results for diffusion coefficient, computed as $D=\lim _{t \rightarrow \infty}\left\langle\Delta r^{2}\right\rangle /(6 t)$, as a function of $d$ are shown in Figure 3 by the solid symbols. For NPs with $d>a$ in

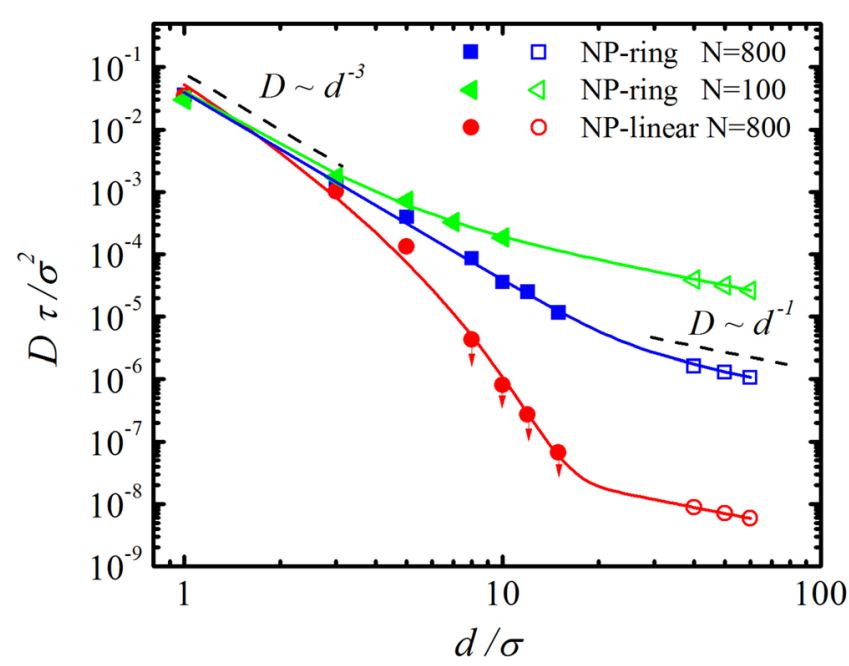

Figure 3. Diffusion coefficients $D$ as functions of diameter $d$ for monomers $(d=1 \sigma)$ and NPs $(d \geq 3 \sigma)$ in ring polymers with $N=800$ (blue squares) and $N=100$ (green triangles) and in linear polymers with $N=800$ (red circles). Solid symbols are results of $D=$ $\lim _{t \rightarrow \infty}\left\langle\Delta r^{2}\right\rangle /(6 t)$. Arrows below the solid symbols indicate that the results are upper bounds. Open symbols are results computed using the Stokes-Einstein relation with slip boundaries between NPs and polymers. Solid lines are best fits to crossover functions.

linear polymers, we can estimate the upper bounds of $D$ (indicated by the arrows in Figure 3). As in previous simulations, ${ }^{4}$ for the sake of consistency, we do not correct $D$ for the long-range hydrodynamic effects associated with the finite sample sizes. ${ }^{44,45}$ In the limit of very large $d$, it is expected that NP mobility is completely coupled to the melt viscosity $\eta$ of polymers, and thus $D=k_{\mathrm{B}} T /(2 \pi \eta d)$ assuming the StokesEinstein relation with slip boundaries between NPs and polymers. The slip lengths for NPs in the simulations are anticipated to be much larger than $d$, resulting in the slip NPpolymer boundaries. Previous simulation results ${ }^{4}$ of NPs in linear polymer melts have also been analyzed using the slip boundary condition. For $d \geq 40 \sigma$, the results of $D$ calculated using the Stokes-Einstein relation are shown in Figure 3 by the open symbols. Results for $\eta$ in simulations are presented in Section 2 of the Supporting Information. Simulation data for the $d$ dependence of $D$ can be extrapolated to match the Stokes-Einstein relation for both NP-ring and NP-linear systems.

For $d<a$, the decrease of $D$ is almost the same for NP-ring and NP-linear systems and exhibits $D \sim d^{-3}$ scaling (see Figure $3)$, which agrees with the theoretical prediction ${ }^{12}$ for the coupling between NP motion and unentangled Rouse dynamics. However, for $d>a$, the decrease is much steeper for NPs in linear chains as the NPs are confined by the entanglement network before they are able to freely diffuse. For $d=15 \sigma$, even the estimated upper bound of $D$ in linear chains is smaller than $D$ in rings with the same $N$ by more than 2 orders of magnitude. Recently, Nahali and Rosa ${ }^{46}$ have compared the motion of NPs in concentrated solutions of semiflexible linear polymers and ring polymers. They observed 
that $D$ for $d \approx a$ is about 3 times larger in ring polymers than in linear polymers. In our simulations, the ratio of MSDs in ring and linear polymers is also $\approx 3$ at $d \approx a$. However, our simulations go further up to $d \approx 3$ and show much stronger effects of polymer architecture on $D$.

There is a crossover of the $d$ dependence of $D$ from $D \sim d^{-3}$ to $D \sim d^{-1}$ for NPs with $d>a$ in linear polymers. The crossover is broad and extends at least up to $d \approx 3 a$. According to Cai et al., ${ }^{12}$ hopping diffusion of NPs with $d$ moderately larger than $a$ results in $D \sim \exp (-d / a)$ that broadens the crossover to the Stokes-Einstein regime. The results of $D$ for NP-linear systems are fitted by a crossover function $D=c_{1}\left(\sigma^{2} / \tau\right)(\sigma / d)^{3}$ $\exp \left(-c_{2} d / a\right)+k_{\mathrm{B}} T /(2 \pi \eta d)$, in which $c_{1}$ and $c_{2}$ are fitting parameters, and the exponential term is motivated by the theory of hopping diffusion. The best-fit result with $c_{1}=0.081$ \pm 0.018 and $c_{2}=2.2 \pm 0.1$ is shown by the red line in Figure 3 (see Figure S2 for the details of fitting).

For NPs with $a<d<R$ in ring polymers, our scaling analysis predicts $D \sim d^{-5}$. This prediction is based on the ansatz that the effective viscosity $\eta_{\text {eff }}$ that a NP with $a<d<R$ experiences in the Fickian regime is equal to the melt viscosity of ring polymers with size $R \approx d$. A ring polymer with $R \approx d$ contains $n$ $\sim d^{3}$ monomers, and the melt viscosity ${ }^{27} \eta \sim n^{4 / 3} \sim d^{4}$. Therefore, $\eta_{\text {eff }} \sim d^{4}$ and $D \sim k_{\mathrm{B}} T /\left(\eta_{\text {eff }} d\right) \sim d^{-5}$. Grosberg et al. $^{43}$ found that the wave vector $q$-dependent effective viscosity $\eta(q) \sim q^{-4}$ for $1 / R<d<1 / a$ in the FLG model. Based on their result, the effective viscosity for Fickian diffusion of a NP with $a<d<R$ is $\eta(q \approx 1 / d) \sim d^{4}$, in agreement with our result $\eta_{\text {eff }} \sim$ $d^{4}$.

The $d$ dependence of $D$ for NP-ring systems with $a<d<R$ in our simulations is weaker than the theoretical prediction $D \sim$ $d^{-5}$. The crossovers between the scaling regime for $a<d<R$ and two neighboring regimes contribute to the weakening of the $d$ dependence. The results of $D$ for NPs in rings with $N=$ 800 and $N=100$ are fitted simultaneously using a crossover function $D=b_{1}\left(\sigma^{2} / \tau\right)(\sigma / d)^{3} /\left(1+b_{2} a^{2} / d^{2}\right)+k_{\mathrm{B}} T /(2 \pi \eta d)$, in which the fitting parameters $b_{1}$ and $b_{2}$ are independent of $N$. The fitting function describes the crossover from $D \sim d^{-3}$ for small NPs coupled to the unentangled dynamics of local ring sections to $D \sim d^{-5}$ for NPs coupled to the self-similar entangled dynamics of rings and then to $D \sim d^{-1}$ in the Stokes-Einstein regime. The best-fit results with $b_{1}=0.039 \pm$ 0.003 and $b_{2}=0.05 \pm 0.03$ are shown in Figure 3 (see Figure S3 for the details of fitting). $D \sim d^{-5}$ dominates over $D \sim d^{-3}$ only for $d>25 \sigma$. This crossover occurs in the regime where $D$ $\sim d^{-1}$ dominates the overall $d$ dependence. Therefore, there is no apparent $D \sim d^{-5}$ regime. For $N=100$, the crossover to $D \sim$ $d^{-1}$ occurs at smaller $d$, and even the $D \sim d^{-3}$ regime is not well developed.

\section{CONCLUSION}

In conclusion, our simulations show that the motion of NPs can be strongly suppressed prior to Fickian diffusion in entangled linear polymers. Such a strong suppression occurs progressively as the NP diameter $d$ becomes increasingly larger than the entanglement spacing $a$. The motion of NPs with $d>$ $a$ in entangled nonconcatenated ring polymers is not strongly suppressed as in entangled linear polymers. The decrease of diffusion coefficient $D$ with increasing $d$ in entangled rings is more gradual compared to the steep reduction of $D$ in entangled linear chains as $d$ exceeds $a$. NP motion for $d>a$ in entangled linear chains is understood through a hopping diffusion mechanism. ${ }^{14}$ For NPs with $d>a$ in entangled rings, we develop a scaling model for the coupling between NP motion and the self-similar dynamics of entangled rings. Our results demonstrate the role of polymer architecture in the dynamical coupling between NPs and polymers in nanocomposites.

\section{ASSOCIATED CONTENT}

\section{S Supporting Information}

The Supporting Information is available free of charge on the ACS Publications website at DOI: 10.1021/acs.macromol.6b02632.

Estimate of the threshold NP diameter $d_{c}$ for the complete coupling between NPs and relaxation dynamics of ring polymers; details about fitting the dependence of diffusion coefficient $D$ on $d$ to a crossover function (PDF)

\section{AUTHOR INFORMATION}

\section{Corresponding Author}

*E-mail: mr@unc.edu (M.R.).

ORCID

Ting Ge: 0000-0003-2456-732X

Notes

The authors declare no competing financial interest.

\section{ACKNOWLEDGMENTS}

M.R. acknowledges financial support from the National Science Foundation under Grants DMR-1309892, DMR-1436201, and DMR-1121107, the National Institutes of Health under Grants P01-HL108808 and 1UH2HL123645, and the Cystic Fibrosis Foundation. This work was performed, in part, at the Center for Integrated Nanotechnologies, an Office of Science User Facility operated for the U.S. Department of Energy (DOE) Office of Science. This research used resources obtained through the Advanced Scientific Computing Research (ASCR) Leadership Computing Challenge (ALCC) at the National Energy Research Scientific Computing Center (NERSC), which is supported by the Office of Science of the U.S. Department of Energy under Contract DE-AC02-05CH11231. Sandia National Laboratories is a multiprogram laboratory managed and operated by Sandia Corporation, a wholly owned subsidiary of Lockheed Martin Corporation, for the U.S. Department of Energy's National Nuclear Security Administration under Contract DE-AC04-94AL85000.

\section{REFERENCES}

(1) Balazs, A. C.; Emrick, T.; Russell, T. P. Nanoparticle polymer composites: Where two small worlds meet. Science 2006, 314, 11071110.

(2) Mackay, M. E.; Dao, T. T.; Tuteja, A.; Ho, D. L.; Van Horn, B.; Kim, H. C.; Hawker, C. J. Nanoscale effects leading to non-Einsteinlike decrease in viscosity. Nat. Mater. 2003, 2, 762-766.

(3) Tuteja, A.; Mackay, M. E.; Hawker, C. J.; Van Horn, B. Effect of Ideal, Organic Nanoparticles on the Flow Properties of Linear Polymers: Non-Einstein-like Behavior. Macromolecules 2005, 38, 8000-8011.

(4) Kalathi, J. T.; Grest, G. S.; Kumar, S. K. Universal Viscosity Behavior of Polymer Nanocomposites. Phys. Rev. Lett. 2012, 109, 198301.

(5) Szymanski, J.; Patkowski, A.; Wilk, A.; Garstecki, P.; Holyst, R. Diffusion and Viscosity in a Crowded Enviroment: from Nano- to Macroscale. J. Phys. Chem. B 2006, 110, 25593-25597. 
(6) Guo, H.; Bourret, G.; Lennox, R. B.; Sutton, M.; Harden, J. L.; Leheny, R. L. Entanglement-Controlled Subdiffusion of Nanoparticles within Concentrated Polymer Solutions. Phys. Rev. Lett. 2012, 109, 055901.

(7) Grabowski, C. A.; Mukhopadhyay, A. Size Effect of Nanoparticle Diffusion in a Polymer Melt. Macromolecules 2014, 47, 7238-7242.

(8) Mangal, R.; Srivastava, S.; Narayanan, S.; Archer, L. A. SizeDependent Particle Dynamics in Entangled Polymer Nanocomposites. Langmuir 2016, 32, 596-603.

(9) Liu, J.; Cao, D.; Zhang, L. Molecular Dynamics Study on Nanoparticle Diffusion in Polymer Melts: A Test of the StokesEinstein Law. J. Phys. Chem. C 2008, 112, 6653-6661.

(10) Kalathi, J. T.; Yamamoto, U.; Schweizer, K. S.; Grest, G. S.; Kumar, S. K. Nanoparticle Diffusion in Polymer Nanocomposites. Phys. Rev. Lett. 2014, 112, 108301.

(11) Wyart, F. B.; de Gennes, P. G. Viscosity at small scales in polymer melts. Eur. Phys. J. E: Soft Matter Biol. Phys. 2000, 1, 93.

(12) Cai, L. H.; Panyukov, S.; Rubinstein, M. Mobility of Nonsticky Nanoparticles in Polymer Liquids. Macromolecules 2011, 44, 78537863.

(13) Yamamoto, U.; Schweizer, K. S. Theory of nanoparticle diffusion in unentangled and entangled polymer melts. J. Chem. Phys. 2011, 135, 224902.

(14) Cai, L. H.; Panyukov, S.; Rubinstein, M. Hopping Diffusion of Nanoparticles in Polymer Matrices. Macromolecules 2014, 48, 847862.

(15) Dell, Z. E.; Schweizer, K. S. Theory of Localization and Activated Hopping of Nanoparticles in Cross-Linked Networks and Entangled Polymer Melts. Macromolecules 2014, 47, 405-414.

(16) Yamamoto, U.; Schweizer, K. S. Microscopic Theory of the Long-Time Diffusivity and Intermediate-Time Anomalous Transport of a Nanoparticle in Polymer Melts. Macromolecules 2015, 48, 152163.

(17) McLeish, T. C. B. Tube theory of entangled polymer dynamics. Adv. Phys. 2002, 51, 1379-1527.

(18) Rubinstein, M.; Colby, R. H. Polymer Physics; Oxford University Press: 2003.

(19) Kapnistos, M.; Lang, M.; Vlassopoulos, D.; Pyckhout-Hintzen, W.; Richter, D.; Cho, D.; Chang, T.; Rubinstein, M. Unexpected power-law stress relaxation of entangled ring polymers. Nat. Mater. 2008, 7, 997-1002.

(20) Halverson, J. D.; Lee, W. B.; Grest, G. S.; Grosberg, A. Y.; Kremer, K. Molecular Dynamics Simulation Study of Nonconcatenated Ring Polymers in a Melt: I. Statics. J. Chem. Phys. 2011, 134, 204904.

(21) Halverson, J. D.; Lee, W. B.; Grest, G. S.; Grosberg, A. Y.; Kremer, K. Molecular Dynamics Simulation Study of Nonconcatenated Ring Polymers in a Melt: II. Dynamics. J. Chem. Phys. 2011, 134, 204905.

(22) Halverson, J. D.; Grest, G. S.; Grosberg, A. Y.; Kremer, K. Rheology of Ring Polymer Melts: From Linear Contaminants to RingLinear Blends. Phys. Rev. Lett. 2012, 108, 038301.

(23) Pasquino, R.; et al. Viscosity of Ring Polymer Melts. ACS Macro Lett. 2013, 2, 874-878.

(24) Gooßen, S.; Braś, A.; Krutyeva, M.; Sharp, M.; Falus, P.; Feoktystov, A.; Gasser, U.; Pyckhout-Hintzen, W.; Wischnewski, A.; Richter, D. Molecular Scale Dynamics of Large Ring Polymers. Phys. Rev. Lett. 2014, 113, 168302.

(25) Rosa, A.; Everaers, R. Ring Polymers in the Melt State: The Physics of Crumpling. Phys. Rev. Lett. 2014, 112, 118302.

(26) Smrek, J.; Grosberg, A. Y. Understanding the dynamics of rings in the melt in terms of the annealed tree model. J. Phys.: Condens. Matter 2015, 27, 064117.

(27) Ge, T.; Panyukov, S.; Rubinstein, M. Self-Similar Conformations and Dynamics in Entangled Melts and Solutions of Nonconcatenated Ring Polymers. Macromolecules 2016, 49, 708-722.

(28) McLeish, T. C. B. Polymer dynamics: Floored by the rings. Nat. Mater. 2008, 7, 933-935.
(29) Michieletto, D.; Turner, M. S. A toplogical driven glass in ring polymers. Proc. Natl. Acad. Sci. U. S. A. 2016, 113, 5195-5200.

(30) Tsalikis, D. G.; Mavrantzas, V. G.; Vlassopoulos, D. Analysis of Slow Modes in Ring Polymers: Threading of Rings Controls LongTime Relaxation. ACS Macro Lett. 2016, 5, 755-760.

(31) Cremer, T.; Cremer, C. Chromosom territories, nuclear architecture and gene regulation in mammalian cells. Nat. Rev. Genet. 2001, 2, 292.

(32) Halverson, J.; Smrek, J.; Kremer, K.; Grosberg, A. Y. From a melt of rings to chromosome territories: the role of topological constraints in genome folding. Rep. Prog. Phys. 2014, 77, 022601.

(33) Obukhov, S. P.; Rubinstein, M.; Colby, R. H. Network Modulus and Superelasticity. Macromolecules 1994, 27, 3191-3198.

(34) Kremer, K.; Grest, G. S. Dynamics of entangled linear polymer melts: A molecular-dynamics simulation. J. Chem. Phys. 1990, 92, 5057.

(35) in 't Veld, P. J.; Plimpton, S. J.; Grest, G. S. Accurate and efficient methods for modeling colloidal mixtures in an explicit solvent using molecular dynamics. Comput. Phys. Commun. 2008, 179, 320.

(36) Meng, D.; Kumar, S. K.; Cheng, S.; Grest, G. S. Simulating the miscibility of nanoparticles and polymer melts. Soft Matter 2013, 9, 5417-5427.

(37) Everaers, R.; Sukumaran, S. K.; Grest, G. S.; Svaneborg, C.; Sivasubramanian, A.; Kremer, K. Rheology and Microscopic Topology of Entangled Polymeric Liquids. Science 2004, 303, 823-826.

(38) Hsu, H.-P.; Kremer, K. Static and dynamic properties of large polymer melts in equilibrium. J. Chem. Phys. 2016, 144, 154907.

(39) Grest, G. S. Communication: Polymer entanglement dynamics: Role of attractive interactions. J. Chem. Phys. 2016, 145, 141101.

(40) Ge, T.; Robbins, M. O.; Perahia, D.; Grest, G. S. Healing of polymer interfaces: Interfacial dynamics, entanglements,and strength. Phys. Rev. E 2014, 90, 012602.

(41) Likhtman, A. E.; McLeish, T. C. B. Quantitative Theory for Linear Dynamics of Linear Entangled Polymers. Macromolecules 2002, 35,6332 .

(42) Kavassalis, T. A.; Noolandi, J. New View of Entanglements in Dense Polymer Systems. Phys. Rev. Lett. 1987, 59, 2674.

(43) Grosberg, A. Y.; Joanny, J.-F.; Srinin, W.; Rabin, Y. ScaleDependent Viscosity in Polymer Fluids. J. Phys. Chem. B 2016, 120, $6383-6390$

(44) Lobaskin, V.; Dunweg, B. A new model for simulating colloidal dynamics. New J. Phys. 2004, 6, 54.

(45) Yeh, I.-C.; Hummer, G. System-Size Dependence of Diffusion Coefficients and Viscosities from Molecular Dynamics Simulations with Periodic Boundary Conditions. J. Phys. Chem. B 2004, 108, 15873.

(46) Nahali, N.; Rosa, A. Density effects in entangled solutions of linear and ring polymers. J. Phys.: Condens. Matter 2016, 28, 065101. 